\title{
No evidence for the involvement of the lipoxin A4 receptor (FPR2/ALX) gene in the susceptibility to coronary artery disease
}

\author{
Vanessa Waechter, Jacqueline Marti-Jaun, \\ Angelika Weber, Zoltan Laszlo Madi and \\ Martin Hersberger* \\ Division of Clinical Chemistry and Biochemistry, \\ University Children's Hospital Zurich and Center for \\ Integrative Human Physiology, University of Zurich, \\ Zurich, Switzerland
}

Keywords: atherosclerosis; FPRL1; FPR2; FPR2/ALX; lipoxin A4 receptor; polymorphism.

The lipoxin A4 receptor (FPR2/ALX) belongs to the class A rhodopsin $\mathrm{G}$ protein-coupled receptor superfamily and plays a role in chemotaxis and activation of phagocytes (1). Several structurally diverse agonistic ligands, including peptides and lipid mediators have been shown to bind to the FPR2/ALX (2). Since the discovery of serum amyloid A (SAA) as a proinflammatory ligand for FPR2/ALX, more peptides were identified, which all mediate a pro-inflammatory activation of leukocytes through FPR2/ALX (1). In contrast, FPR2/ALX can also bind anti-inflammatory ligands, such as lipoxin A4 (LXA4) and annexin-1 (2). Indeed, LXA4 was shown to inhibit neutrophil migration, to induce chemotaxis of monocytes and to promote the non-phlogistic phagocytosis of apoptotic neutrophils by macrophages (2). Thus, FPR2/ALX might induce anti-inflammatory effects when pro-resolving mediators are present.

Expression of the FPR2/ALX has been observed in several human chronic inflammatory diseases, such as dermatitis, rheumatoid arthritis, and severe asthma, however, the role of the FPR2/ALX in atherosclerosis has not been investigated (3).

Atherosclerosis is a multifactorial disease that is characterized by chronic inflammation at every stage with leukocytes infiltrating the arterial intima (4). To investigate the role of FPR2/ALX in atherosclerosis we screened the

\footnotetext{
*Corresponding author: Martin Hersberger, Division of Clinical Chemistry and Biochemistry, University Children's Hospital Zurich, Steinwiesstr. 75, 8032 Zurich, Switzerland Phone: +41-44-266-75-41, Fax: +41-44-266-71-69, E-mail: martin.hersberger@kispi.uzh.ch Received June 14, 2011; accepted September 9, 2011; previously published online October 6, 2011
}

FPR2/ALX gene for polymorphisms and analyzed whether the detected polymorphisms are associated with coronary artery disease (CAD) in a case-control study involving 497 Caucasians $(5,6)$. We initially screened the promoter, the coding region and the $3^{\prime}$ region of the FPR2/ALX gene of 98 Caucasians for polymorphisms by DHPLC and detected eight variations, five of which were located in the promoter region, one in the coding region, and two in the $3^{\prime}$ region (Table 1). Seven of these variations proved to be polymorphisms with minor allele frequencies between $5 \%$ and $32 \%$, while variant c.410G $>$ A was a private mutation. The genotype frequency for all polymorphisms was in agreement with those predicted by the Hardy-Weinberg equilibrium in the case-control sample.

This case-control sample for CAD included 259 patients with $>50 \%$ stenosis in at least one coronary artery and 238 subjects without a history of CAD, stroke, or peripheral vascular disease $(5,6)$. Written informed consent was obtained from all participants and the Local Ethics Committee approved the study. The case-control study has a power of $25 \%-80 \%$ to detect an OR of 1.7 for minor allele frequencies of $5 \%-30 \%$.

Logistic regression analysis revealed no association between five polymorphisms of the FPR2/ALX gene and CAD (Table 1), while the $\mathrm{T}$ allele of the c.-7893C $>\mathrm{T}$ polymorphism was associated with an increased risk for CAD ( $\mathrm{OR}=2.9$; confidence interval 1.2-7.1; p-value: 0.018). This c. $-7893 \mathrm{C}>\mathrm{T}$ polymorphism is located in the core promoter of the FPR2/ALX gene and could therefore modify the binding site for a transcription factor and hence alter transcription of the gene (8).

To test whether the $\mathrm{C}>\mathrm{T}$ transition affects the transcriptional activity of the promoter, we subcloned the FPR2/ALX core promoter upstream of a luciferase reporter gene and introduced all the variations of the minor alleles of the three promoter polymorphisms into individual constructs. These constructs were then transiently transfected into THP-1 macrophages and the transcriptional activity of the different promoter alleles was judged from the intensity of the luciferase reporter enzyme (Figure 1, dual-luciferase reporter assay). None of the three polymorphisms c.-7978A $>$ G, c. $-7986 A>G$ and c. $-7893 \mathrm{C}>\mathrm{T}$ altered the transcriptional activity of the FPR2/ALX promoter in macrophages, suggesting that the observed association of the SNP c.-7893C $>$ T with CAD was rather a chance finding than a real association. This is 
Table 1 Logistic regression model predicting CAD status for cases.

\begin{tabular}{|c|c|c|c|c|c|c|c|}
\hline \multirow{2}{*}{$\begin{array}{l}\text { NM01005738 } \\
\text { c. }-8751 \mathrm{~A}>\mathrm{G} \\
\text { rs } 11666254\end{array}$} & \multicolumn{2}{|c|}{ Allele frequency, $\%$} & \multirow{2}{*}{$\begin{array}{l}\text { Genotype controls, \% } \\
47.3 / 43.9 / 8.9\end{array}$} & \multirow{2}{*}{$\begin{array}{l}\text { Genotype cases, \% } \\
47.5 / 41.2 / 11.3\end{array}$} & \multirow{2}{*}{$\frac{\mathrm{OR}}{1.72}$} & \multirow{2}{*}{$\begin{array}{l}95 \% \mathrm{CI} \\
0.75-3.94\end{array}$} & \multirow{2}{*}{$\frac{\mathrm{p} \text {-Value }}{0.20}$} \\
\hline & A: 68.6 & G: 31.4 & & & & & \\
\hline $\begin{array}{l}\text { c. }-8461 T>G \\
\text { rs } 11670660\end{array}$ & $\mathrm{~T}: 68.3$ & G: 31.7 & 46.4/43.9/9.7 & 47.5/41.6/10.9 & 0.60 & $0.26-1.36$ & 0.22 \\
\hline $\begin{array}{l}\text { c. }-7986 A>G \\
\text { rs } 35215887\end{array}$ & A: 73 & G: 27 & $49.8 / 46 / 4.2$ & $52.1 / 42 / 5.8$ & 0.98 & $0.69-1.40$ & 0.92 \\
\hline $\begin{array}{l}\text { c. }-7978 A>G \\
\text { rs } 35225016\end{array}$ & A:95 & G: 5 & $92.4 / 7.6 / 0$ & $89.1 / 9.7 / 1.2$ & 1.42 & $0.75-2.68$ & 0.29 \\
\hline $\begin{array}{l}\text { c. }-7893 \mathrm{C}>\mathrm{T} \\
\text { rs } 56238033\end{array}$ & C: 88.2 & T: 11.8 & $77.2 / 22.8 / 0$ & 77.4/20.6/1.9 & 2.93 & $1.20-7.13$ & 0.02 \\
\hline $\begin{array}{l}\text { c. } 410 \mathrm{G}>\mathrm{A} \\
\text { c. } 1315 \mathrm{~A}>\mathrm{G} \\
\mathrm{rs} 17695052\end{array}$ & A: 89.1 & G: 10.9 & $76.8 / 23.2 / 0$ & $\begin{array}{l}\text { Private mutation } \\
80.5 / 18.3 / 1.2\end{array}$ & 0.66 & $0.19-2.28$ & 0.51 \\
\hline $\begin{array}{l}\text { c. } 1346 C>T \\
\text { rs17695064 }\end{array}$ & C: 89.4 & T: 10.6 & $77.2 / 22.4 / 0.4$ & 81.3/17.9/0.8 & 0.51 & $0.17-1.58$ & 0.24 \\
\hline
\end{tabular}

DNA extracted from EDTA-supplemented blood was genotyped by tetra-primer analysis for each variation (7). Association with CAD was analyzed by logistic regression using an additive genetic model adjusted for age and sex. $\mathrm{p}<0.05$.

supported by the skewed distribution of the homozygous TT genotypes between cases and controls in the study sample. While the TT genotype was observed to a low frequency in cases, this genotype was absent in controls $(1.95 \%$ vs. $0 \%$, respectively). The FPR2/ALX haplotypes derived from the seven polymorphisms also showed no association with CAD.

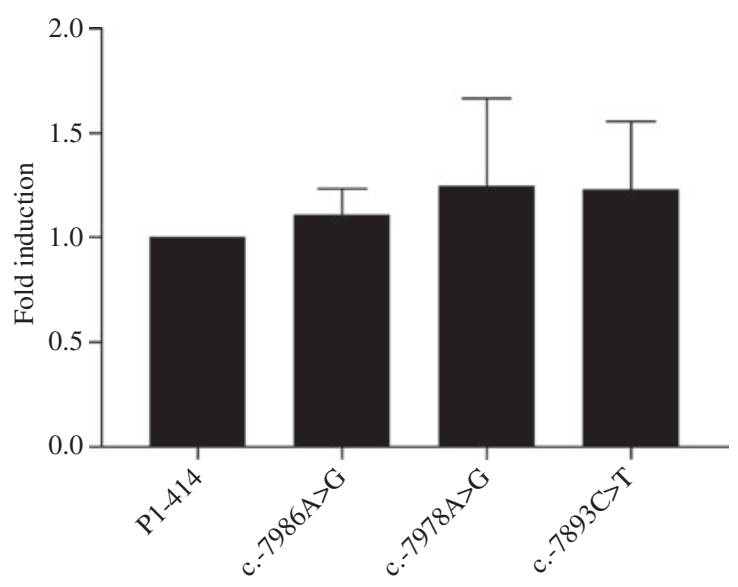

Figure 1 Basal transcription of the different FPR2/ALX core promoter alleles observed.

Luciferase expression of the four FPR2/ALX core promoter alleles was determined (c.-7986A $>$ G: A-to-G base exchange at position -7986; c.-7978A >G: A-to-G base exchange at position -7978; c.7893C $>$ T: C-to-T base exchange at position -7893). Human THP-1 monocytes were transfected with the constructs mentioned and a renilla control construct, differentiated into macrophages and the luciferase activity was measured and normalized against the activity of the renilla luciferase $48 \mathrm{~h}$ later. Results were calculated as fold induction over the FPR2/ALX wild-type core promoter. All experiments were done in triplicate and bars indicate the means of three independent experiments with three plasmid preparations. Values represent fold induction compared to the P1-414 vector and the error bars represent the standard deviation $( \pm \mathrm{SD})$.
We observed six haplotypes with a frequency $>1 \%$ which summed up to $94 \%$ of all haplotypes in the sample (data not shown).

In this study, we identified seven polymorphisms in the promoter and in the $3^{\prime}$ region of the FPR2/ALX gene, but no polymorphism was detected in the coding region of the gene. Such a lack of polymorphisms in the coding region of the FPR2/ALX gene has previously been observed in a small study comparing the number of polymorphisms in the coding region between the FPR2/ALX and the closely related FPR1 gene (9). This study found that the two genes originated from a common gene but that they had undergone markedly different evolutionary events since then. While seven polymorphisms have been detected in the coding region of the FPRl gene in this small Caucasian, Black and Asian sample, no polymorphism was detected in the FPR2/ALX coding region in the same racial groups (9). Our study supports and expands these findings, because we screened an additional 196 Caucasian alleles and did not find a polymorphism in the coding region of the FPR2/ALX. Such an absence of functional polymorphisms in the coding region and the lack of functional polymorphisms in the core promoter region suggest that the FPR2/ALX gene is highly conserved in humans and that it may be important for the correct function of leukocytes in innate immunity.

In conclusion, the FPR2/ALX gene was not associated with CAD in our small case-control study, however, our study suggests that the FPR2/ALX gene is conserved in humans, since no polymorphism in the coding region and no functional polymorphisms in the core promoter region have been identified.

\section{Acknowledgments}

This work was supported by grants from the Swiss National Science Foundation and the Hermann Klaus-Stiftung. 


\section{Conflict of interest statement}

Authors' conflict of interest disclosure: The authors stated that there are no conflicts of interest regarding the publication of this article. Research support played no role in the study design; in the collection, analysis, and interpretation of data; in the writing of the report; or in the decision to submit the report for publication.

Research funding: None declared.

Employment or leadership: None declared.

Honorarium: None declared.

\section{References}

1. Ye RD, Boulay F, Wang JM, Dahlgren C, Gerard C, Parmentier $\mathrm{M}$, et al. International union of basic and clinical pharmacology. Lxxiii. Nomenclature for the formyl peptide receptor (fpr) family. Pharmacol Rev 2009;61:119-61.

2. Chiang N, Serhan CN, Dahlen SE, Drazen JM, Hay DW, Rovati GE, et al. The lipoxin receptor alx: potent ligand-specific and stereoselective actions in vivo. Pharmacol Rev 2006;58:463-87.

3. Hersberger M. Potential role of the lipoxygenase derived lipid mediators in atherosclerosis: leukotrienes, lipoxins and resolvins. Clin Chem Lab Med 2010;48:1063-73.
4. Paoletti R, Gotto AM Jr, Hajjar DP. Inflammation in atherosclerosis and implications for therapy. Circulation 2004;109: III20-6.

5. Hersberger M, Muntwyler J, Funke H, Marti-Jaun J, Schulte H, Assmann G, et al. The cag repeat polymorphism in the androgen receptor gene is associated with hdl-cholesterol but not with coronary atherosclerosis or myocardial infarction. Clin Chem 2005;51:1110-5.

6. Wittwer J, Bayer M, Mosandl A, Muntwyler J, Hersberger M. The c.-292c >t promoter polymorphism increases reticulocyte-type 15-lipoxygenase- 1 activity and could be atheroprotective. Clin Chem Lab Med 2007;45:487-92.

7. Hersberger M, Marti-Jaun J, Rentsch K, Hanseler E. Rapid detection of the cyp $2 \mathrm{~d} 6 * 3$, cyp $2 \mathrm{~d} 6 * 4$, and cyp $2 \mathrm{~d} 6 * 6$ alleles by tetraprimer pcr and of the cyp2d6*5 allele by multiplex long pcr. Clin Chem 2000;46:1072-7.

8. Wittwer J, Marti-Jaun J, Hersberger M. Functional polymorphism in alox 15 results in increased allele-specific transcription in macrophages through binding of the transcription factor spil. Hum Mutat 2006;27:78-87.

9. Sahagun-Ruiz A, Colla JS, Juhn J, Gao JL, Murphy PM, McDermott DH. Contrasting evolution of the human leukocyte $\mathrm{n}$-formylpeptide receptor subtypes fpr and fprl1r. Genes Immun 2001;2:335-42. 